\title{
Comunicação LoRa na Zona Rural: Análise Baseada em uma Campanha de Medição no Noroeste Paulista
}

\author{
Alex R. Facina, Raul G. Tancredo, Michelle S. P. Facina e Gustavo Fraidenraich
}

\begin{abstract}
Resumo-Os requisitos de baixo consumo de energia e ampla cobertura de área fazem do Long Range (LoRa) uma ótima candidata para concepção do conceito de Internet of Things. Apesar de já existirem muitos trabalhos publicados nessa área, nenhum deles aborda a realidade brasileira de ser um grande produtor agrícola. Nosso projeto tem como objetivo desenvolver um protótipo de rastreamento bovino de baixo custo usando tal tecnologia de forma a fomentar a agricultura de precisão e aumentar a produtividade do campo. Este artigo apresenta os estudos preliminares acerca do alcance da comunicação na zona rural. Através da análise dos dados, nós obtemos o coeficiente de decaimento e concluímos que o ambiente é adequado para a propagação de sinais LoRa desde que haja linha de visada entre os dispositivos transmissor e receptor.
\end{abstract}

Palavras-Chave-5G, LoRa, propagação, protótipo, rastreamento bovino.

Abstract-Low power consumption requirements and comprehensive area coverage make LoRa a great candidate for designing the Internet of Things concept. Although there are already many works published in this area, none of them address the Brazilian reality of being a large agricultural producer. Our project aims to develop a low-cost bovine tracking prototype using such technology to foster precision agriculture and increase field productivity. This article presents preliminary studies on the scope of communication in rural areas. Through data analysis, we obtain the decay coefficient and conclude that the environment is suitable for the propagation of LoRa signals as long as there is the lineof-sight between transmitter and receiver devices.

Keywords-5G, LoRa, propagation, prototype, cattle tracking.

\section{INTRODUÇÃO}

Desde meados do século XVIII, o mundo já passou por três revoluções industriais. A primeira delas é caracterizada pela transição da produção manual à mecanizada. A segunda, por volta de 1850, foi alavancada graças à eletricidade e permitiu a produção em larga escala. A terceira, já no século XX, aconteceu com a chegada da eletrônica, da tecnologia da informação e das telecomunicações [1].

Atualmente, nós estamos vivendo a quarta revolução industrial. Também conhecida como Indústria 4.0, ela é sinônimo de manufatura inteligente, em que a transformação digital passa a englobar não só centros urbanos. Quando aplicada em zonas rurais, a chamada agricultura de precisão tem como objetivo unir novas tecnologias à

Alex Facina, Raul Tancredo, Michelle Facina e Gustavo Fraidenraich fazem parte do Departamento de Comunicações (DECOM) da Faculdade de Engenharia Elétrica e Computação da Universidade Estadual de Campinas, Campinas-SP, Brasil. e-mail: alexfacina@gmail.com preocupação de minimizar desperdícios, com uma produção sustentável, ou seja, maior produtividade, flexibilidade e agilidade [2].

E é nesse contexto que surge o conceito de Internet of Things (IoT) no ramo de comunicações sem fio. Basicamente, a ideia consiste em entidades físicas conectadas e comunicando-se entre si e com o usuário através de sensores inteligentes e softwares que transmitem dados para uma rede. Essas entidades são conectadas à internet através de protocolos de comunicação sem fio atuais, como Bluetooth e Wi-Fi. Mas vale ressaltar que, embora eles ofereçam suporte a altas taxas de dados, não conseguem atender aos requisitos de baixo consumo de energia e ampla cobertura de área [3].

É bem verdade que a próxima geração de redes celulares (5G) será capaz de fornecer alta velocidade e estabilidade para as suas redes. No entanto, a previsão para sua instalação e funcionamento efetivo ainda pode demorar. O leilão de $5 \mathrm{G}$ no Brasil prevê que tal tecnologia estará disponível em todas as capitais em 2022. Mas não há prazo delimitado para atender regiões menos populosas, como grande parte das propriedades agrícolas.

Dentre as tecnologias que precedem ou até mesmo concorrem com as redes $5 \mathrm{G}$ na transformação digital agrícola, as redes do tipo Low Power Wide Area Network (LPWAN) surgiram para conectar milhares de coisas de menor valor agregado a redes de melhor cobertura, menor custo de conexão e menor consumo de bateria. Dentre as redes LPWAN existentes, uma das tecnologias que tem se destacado comercialmente é a tecnologia Long Range (LoRa) com seu protocolo LoRaWAN. Criada na França em 2009 e adquirida pela Semtech em 2012, ela engloba grandes companhias que têm em comum o interesse no desenvolvimento e na aplicação da rede LoRa, garantindo a interoperabilidade de todos os seus produtos.

As redes LoRaWAN usam bandas de frequência Industrial, Sientific, and Medical (ISM) não-licenciadas. Por exemplo, na Europa elas operam na banda de 867-869 $\mathrm{MHz}$ enquanto no Brasil sua atuação é de 902-907,5 $\mathrm{MHz}$ e 915-928 MHz, conforme ato 14448 da Anatel [4]. Graças ao espalhamento espectral, ela é mais resistente a interferências e sua potência é muito menor do que outras tecnologias. Em alguns casos sua sensibilidade de recepção é até abaixo do nível de ruído. Isso significa que mesmo com baixa intensidade do sinal recebido ou do inglês Received Signal Strength Indicator (RSSI), os dispositivos LoRaWAN são capazes de receber e decodificar a mensagem [5].

Sobre essa tecnologia, a maioria dos trabalhos publica- 
dos na literatura dizem respeito a estudos desenvolvidos em outros países. Por exemplo, Augustin et al. [6] avaliam o desempenho da camada física e de enlace através de testes de campo e simulações na França. Entre alguns parâmetros que podem ser ajustados eles destacam o fator de espalhamento (spreading factor (SF), no inglês) que está diretamente relacionado ao número de bits efetivos codificados por símbolo e a sua resistência a interferência do ruído. Quanto maior o SF, mais robusta é a transmissão. No entanto, isso significa também que a portadora é mantida por mais tempo no ar, fazendo com que o dispositivo consuma mais potência.

Por outro lado, uma campanha de medição feita na cidade de Oulu, Finlândia [7] em que o dispositivo final (End Device do inglês) está sob um carro ou um barco, ambos em movimento, revela que para a banda ISM de 868 $\mathrm{MHz}, 14 \mathrm{dBm}$ de potência de transmissão e SF máximo, o alcance de comunicação foi de mais de $15 \mathrm{~km}$ no solo e perto de $30 \mathrm{~km}$ na água. Além disso, o artigo também apresenta um modelo de atenuação de canal a partir dos dados de medição. Já Yim et al. [8] fornece uma análise sobre o desempenho dos parâmetros da camada física das redes LoRa numa floresta de Indiana, Estados Unidos. Eles concluem que o alcance de comunicação LoRa foi menor do que o teoricamente esperado, houve consistência entre a potência do sinal recebida relatada pelos rádios e os dados reais e que a zona de Fresnel afeta as redes LoRa.

O objetivo principal do projeto iniciado há mais de dois anos é conhecer a fundo a tecnologia e aplicá-la ao ambiente rural. Inicialmente, a ideia é desenvolver um protótipo de rastreamento bovino com baixo custo e que seja adequado à realidade brasileira. Seu uso poderá evitar furto de gado, determinar a distância percorrida pelo animal, a fim de estimar a quantidade de ração disponibilizada diariamente, além de poder transmitir informações acerca da saúde do animal, como por exemplo, a temperatura, e antecipar o atendimento veterinário. Por outro lado, tal tecnologia pode ser também usada no sistema de controle de bombas hidráulicas, monitoramento de níveis de bebedouros, rastreamento de insumos de alto custo, entre outros, formando um ecossistema de gerenciamento remoto. Vale ressaltar que o pedido de patente deste produto já se encontra em fase de submissão.

Este artigo faz parte de um estudo de caso prático feito na zona rural do noroeste paulista. Nosso foco aqui é descrever todas as etapas de desenvolvimento do nosso produto e também avaliar o alcance atingido da tecnologia LoRa, que é a camada física do protocolo LoraWan, levando em consideração as características de propagação do sinal no cenário em que há menor consumo de energia. Além disso, com os dados obtidos nas medições, aplicamos o algoritmo de otimização de Levenberg-Marquardt para obter o expoente de decaimento que melhor modela o meio de propagação. Baseado no conhecimento dos autores, esta é a primeira vez que tal análise é feita na zona rural brasileira. Em [9], Ugarte et al. mostram resultados baseados numa campanha de medição feita no ambiente urbano da Universidade Estadual de Campinas, que abrange menor alcance.

O trabalho é organizado da seguinte forma: a Seção II descreve o setup de medição e os algoritmos utilizados, enquanto a Seção III apresenta algumas análises feitas a partir dos dados aquisitados. Finalmente, a Seção IV discorre sobre nossas conclusões e trabalhos futuros.

\section{Setup de MediçÃo}

Nesta seção é feito o detalhamento da composição e funcionamento do sistema para a realização das medições de RSSI em função da distância. Além disso, será apresentado de forma simplificada o algoritmo utilizado para gerar, transmitir, receber e tratar os dados utilizados no processo.

A topologia do sistema utilizado é ponto-a-ponto, composto basicamente por dois transceptores LoRa SX1276. Um deles é móvel e denomina-se End Device, enquanto o outro, foi instalado em um ponto fixo e é referido como Gateway. Ambos são melhor descritos abaixo.

\section{- End Device:}

Dispositivo portátil movido a bateria. Ele é composto por uma placa de desenvolvimento cujos principais componentes são: microcontrolador, receptor de GPS, rádio transceptor LoRa e gerenciador de energia. $\mathrm{O}$ diagrama unifilar da placa e as comunicações utilizadas são mostrados na Fig. 1. O conjunto JB-01 da Tabela I mostra a especificação de cada componente. Dois End Devices foram desenvolvidos. Um com invólucro à prova de intempéries (IP67) adaptado ao cabresto de uma égua, e outro sem o invólucro, com display Organic Light-Emitting Diode (OLED) de $0,96 "$ e fixado numa bicicleta ou no teto de um carro. Sua função é receber, processar e transmitir para o Gateway sua coordenada geográfica recebida pela rede GPS. Ou seja, o receptor Global Positioning System (GPS) recebe a coordenada geográfica e fornece ao microcontrolador através da comunicação Receptor/Transmissor Universal Assíncrono (UART), a sentença em formato National Marine Electronics Association (NMEA). O microcontrolador extrai dessa sentença a latitude, longitude, altitude e envia via Serial Peripheral Interface SPI para o transceptor LoRa fazer a transmissão do pacote. Esse processo foi configurado para repetir a cada dois segundos. O fluxograma do algoritmo, mostrado na Fig. 2, foi desenvolvido em $\mathrm{C} / \mathrm{C}++$ na ferramenta PlatformIO no editor VSCode da Microsoft.

- Gateway:

É composto por um minicomputador de baixo custo, um rádio transceptor LoRa, uma fonte de alimentação 127VAC/5VDC e uma antena, como mostra o diagrama unifilar da Fig. 3. A especificação dos componentes utilizados é mostrada no conjunto JB-02 da Tabela I. Ele foi instalado em um poste de energia elétrica, a 5 metros de altura em relação ao solo na propriedade rural Sítio São Francisco localizada no município de Pedranópolis (SP). Sua função é receber o sinal enviado pelo End Device através do transceptor LoRa de mesmo modelo, avaliar se o pacote está íntegro, extrair as coordenadas geográficas e informações de RSSI e Relação Sinal Ruído (SNR) do pacote, fazer o cálculo da distância ao End Device, adicionar o timestamp, concatenar os dados 


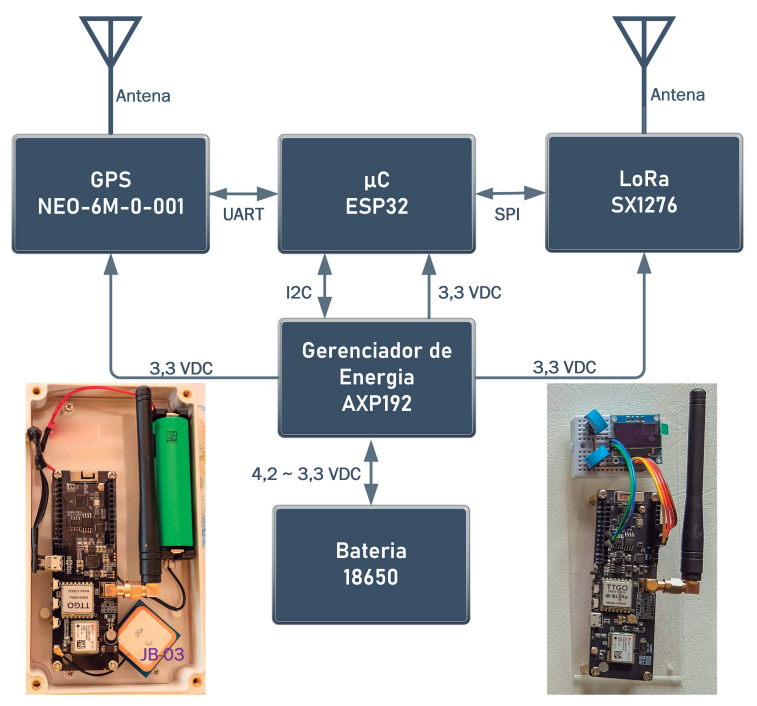

Fig. 1. Diagrama unifilar do End-Device.
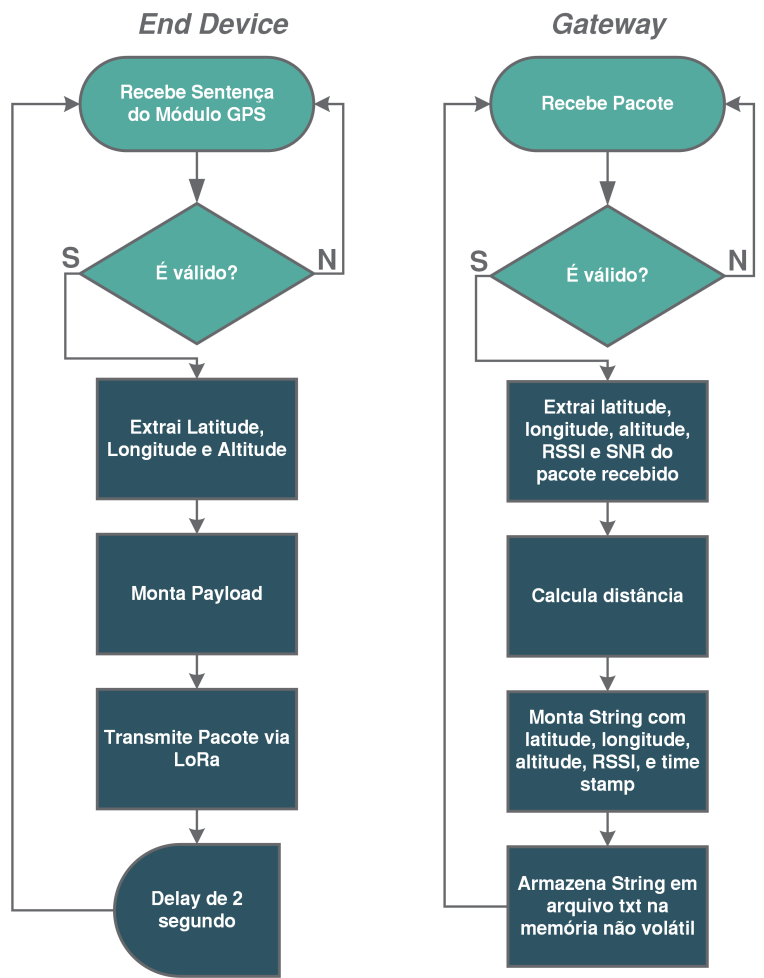

Fig. 2. Algoritmos implementados no End-Device e Gateway.

em uma string e armazenar o arquivo em formato .txt no cartão SD do minicomputador Raspberry Pi. O fluxograma do algoritmo, desenvolvido em Python, é representado na Fig. 2. Vale ressaltar que o Gateway é ligado através da porta Ethernet ao roteador conectado à internet graças a um modem $4 \mathrm{G}$ que permite $\mathrm{o}$ acesso remoto. Esses componentes foram instalados em outra caixa na parte inferior do poste para facilitar a conexão de laptop, o ajuste das configurações do sistema e o troubleshooting. Suas especificações são descritas pelo conjunto JB-03 da Tabela I.

\section{ANÁlise NumÉRICA}

Esta seção descreve como foram gerados os dados das medições de RSSI em função da distância entre o End

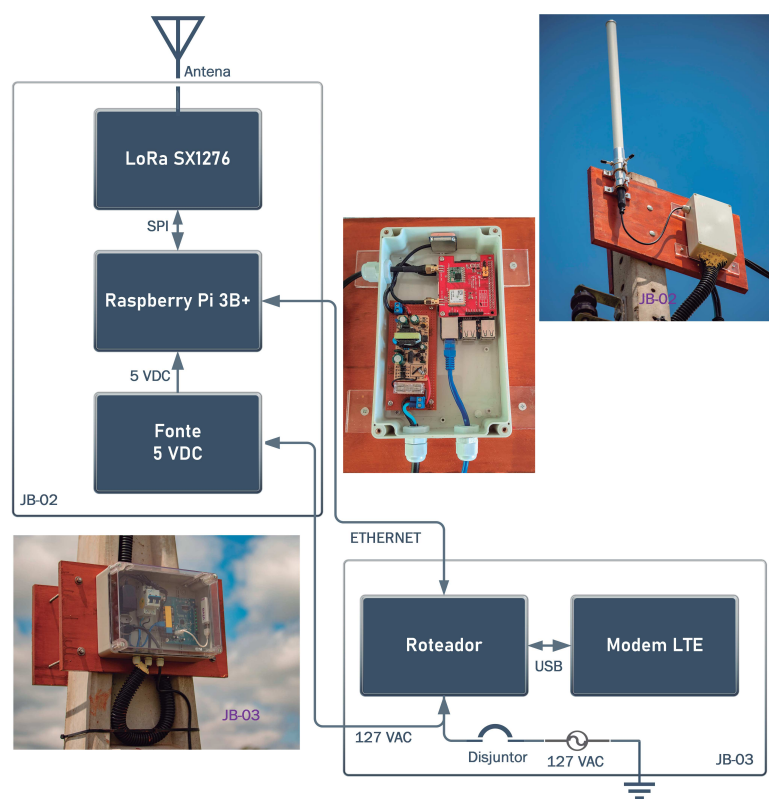

Fig. 3. Diagrama unifilar do Gateway instalado no poste de energia elétrica.

Device e o Gateway. A partir dos parâmetros configurados nos transceptores, como mostrado na Tabela II, foram aquisitadas um total de 23771 amostras. Os transceptores foram configurados de forma que o consumo de energia do End Device seja baixo. Dessa forma, configuramos o SF igual a 7 para que o tempo de transmissão seja menor. Vale a pena também mencionar que a campanha de medição durou quatro meses (entre verão e outono) e levou em consideração diferentes condições de propagação do sinal. Além disso, nós fizemos a média de RSSI para as medições feitas a uma mesma distância. Por fim, nós empregamos a técnica de otimização de LevenbergMarquardt para encontrar o expoente de decaimento.

Os dados de RSSI em função da distância foram gerados adaptando o End Device de três formas: preso a égua, bicicleta e carro, conforme fixação mostrada na Fig. 4. O raio de atuação foi dividido da seguinte forma:

- de 0 a 0,6 km, o End Device foi acoplado no cabresto da égua com livre circulação no pasto;

- de 0,6 a 5 km, o End Device foi acoplado no guidão da bicicleta que percorreu as estradas vicinais do município;

- de 5 a 8,5 km, o End Device foi acoplado no teto do carro, que percorreu estradas vicinais e rodovias que conectam os municípios vizinhos.

O status do sinal durante o percurso feito pelo carro é mostrado na Fig. 5, em que o marcador na cor azul simboliza a recepção do pacote pelo Gateway representado pelo marcador de cor amarelo e o vermelho, pontos em que o sinal do End Device não foi recebido, por estar sob obstrução da zona de Fresnel. Isso fica ainda mais claro quando analisamos o perfil de elevação das duas regiões destacadas com círculo mostrado na Fig. 6. O ponto A mesmo estando situado a $8 \mathrm{~km}$ do Gateway, recebeu sinal enquanto o mesmo não ocorreu para o ponto $\mathrm{B}$ a $4 \mathrm{~km}$.

A partir de uma derivação da fórmula de Friis [10], nós podemos relacionar RSSI, $P_{r x}$, e expoente de decaimento, 
TABELA I

LISTA DE EQUIPAMENTOS UTILIZADOS.

\begin{tabular}{|c|c|c|c|}
\hline & EQUIPAMENTO & FABRICANTE & MODELO \\
\hline \multirow{5}{*}{ JB-01 } & Módulo ESP32 LoRa GPS & TTGO & T-Beam v1.1 \\
\hline & Bateria 18650 & Sony & Vtc6 $3000 \mathrm{mAh}$ \\
\hline & Antena GPS & Sz Beitian Communication & BT-25A \\
\hline & Antena Rubber & G-NiceRF & SW915-WT100 - 915MHz - $3.0 \mathrm{dBi}$ \\
\hline & Caixa de Junção & Wenzhou Hansel Electric Co & 158X90X40mm - IP67 \\
\hline \multirow{5}{*}{ JB-02 } & Raspberry Pi & Raspberry Pi & 3 B plus \\
\hline & Sheild Lora/GPS & Dragino & LoRa/GPS Hat for Rpi v1.4 \\
\hline & Antena Omnidirecional & SteelBras & AP10249 - 900 a $960 \mathrm{MHz}-8.15 \mathrm{dBi}$ \\
\hline & Cabo RF 50 Ohms & Lasershield & N Macho - SMA Macho $50 \mathrm{~cm}$ \\
\hline & Caixa de Junção & Wenzhou Hansel Electric Co & 200x120x55mm - IP67 \\
\hline \multirow{5}{*}{ JB-03 } & Roteador $3 \mathrm{G} / 4 \mathrm{G}$ & D-Link & DWR-116 \\
\hline & Modem 4G & Electroson & LU11 \\
\hline & Disjuntor Bipolar $10 \mathrm{~A}$ & Lukma & DZ47-63-C10 \\
\hline & Extensão Elétrica & Davena & 3 Tomadas $10 \mathrm{~A}$ \\
\hline & Caixa de Junção & Steck & $300 \times 220 \times 120 \mathrm{~mm}-$ IP55 \\
\hline
\end{tabular}

TABELA II

PARÂMETROS CONFIGURADOS NOS TRANSCEPTORES LORA.

\begin{tabular}{l|l}
\hline Largura de Banda & $125 \mathrm{KHz}$ \\
\hline SF & 7 \\
\hline CRC & desabilitado \\
\hline Coding Rate & $4 / 5$ \\
\hline Frequencia de Operação, $f$ & $915 \mathrm{MHz}$ \\
\hline Potência de Transmissão, $P_{t x}$ & $20 \mathrm{dBm}$ \\
\hline Ganho da Antena do End Device, $G_{t x}$ & $3 \mathrm{dBi}$ \\
\hline Ganho da Antena do Gateway, $G_{r x}$ & $8,15 \mathrm{dBi}$ \\
\hline Perda Cabo e conectores, $L_{c a b o}$ & $1 \mathrm{~dB}$ \\
\hline
\end{tabular}

$\gamma$, como

$$
\begin{aligned}
P_{r x}= & P_{t x}+G_{t x}+G_{r x} \\
& -L_{\text {cabo }}+10 \log _{10}\left(\frac{\lambda}{4 \pi}\right)^{2}-10 \gamma \log _{10} d,
\end{aligned}
$$

em que $P_{t x}, G_{t x}$ e $G_{r x}$ representam a potência de transmissão, o ganho da antena do transmissor e o ganho da antena do receptor, respectivamente. Por sua vez, $L_{\text {cabo }}$ é a perda de $1 \mathrm{~dB}$ no cabo e conectores, $d$ é a distância em metros entre End Device e Gateway, enquanto o comprimento de onda é dado por $\lambda=\frac{c}{f}$.

Como em [11], nós ajustamos as medições a uma curva aplicando a técnica de otimização de LevenbergMarquardt [12]. Essa por sua vez, combina as técnicas de Gauss-Newton e de gradiente descendente para convergir mais rápido à solução ótima. Nessa etapa, apenas amostras com $d \leq 3 \mathrm{~km}$ foram consideradas já que para distâncias acima desse valor o limite mínimo de recepção de -120 $\mathrm{dBm}$ foi atingido. $\mathrm{O}$ resultado é mostrado na Fig. 7, em que os dados medidos são representados na cor preta e a curva exponencial ajustada a eles, na cor azul. Dessa forma, é possível encontrar o expoente de decaimento mínimo que satisfaz (1) como $\gamma=3.4108$. Como já era esperado, podemos concluir que a zona rural é adequada para a transmissão de sinais LoRa. Isso ocorre principalmente pela ausência da dispersão de sinais e muitas reflexões. A título de comparação, o resultado obtido no campus da universidade indiana [11] foi $\gamma=5$.616. Lá, o meio de propagação não é tão favorável devido às construções.

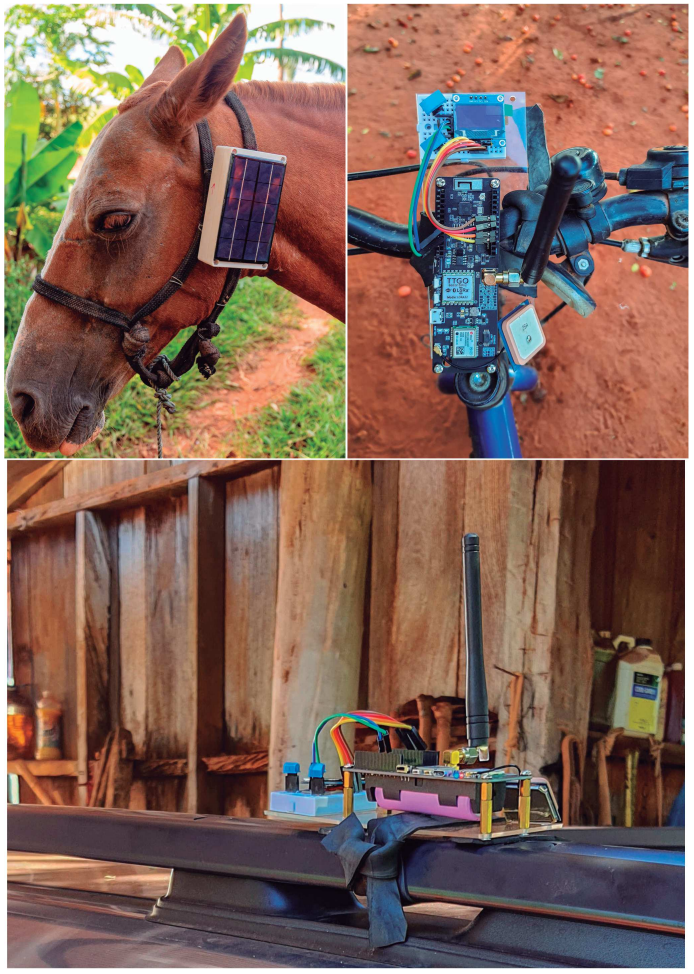

Fig. 4. End Device adaptado a égua, bicicleta e carro.

\section{CONCLUSÃO}

A fim de preencher uma lacuna na literatura, esse artigo apresentou resultados preliminares da camada física do protocolo LoRaWAN na zona rural brasileira. Com nosso protótipo de rastreamento animal de menor custo, nós concluímos que tal tecnologia atinge um alcance de até $8 \mathrm{~km}$ com SF igual a 7 desde que o Gateway esteja com linha de visada ao End Device desobstruída, com coeficiente de decaimento em torno de 4.

Como trabalhos futuros, pretende-se expandir a campanha de medição para a topologia estrela, em que haja um único Gateway concentrador LoraWan SX1301 e alguns End Devices, todos conectados a um servidor. É preciso avaliar o desempenho para diferentes valores de SF e levar em consideração a taxa de erro do pacote, 


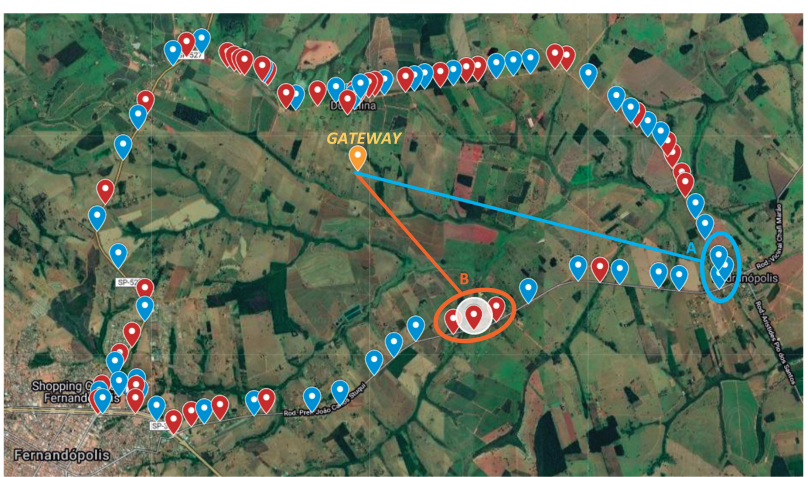

Fig. 5. Status do sinal enviado pelo End Device adaptado no carro.

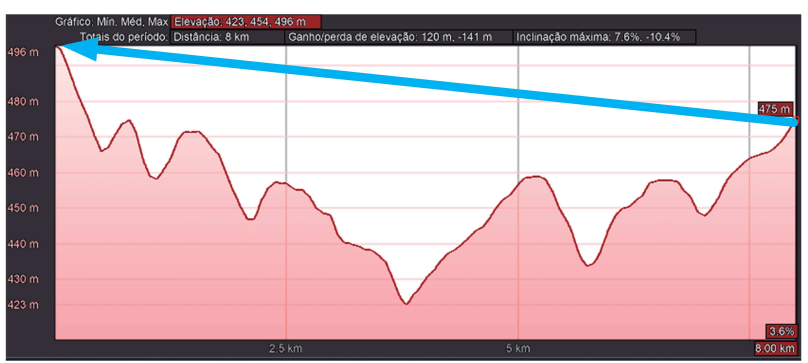

(a)

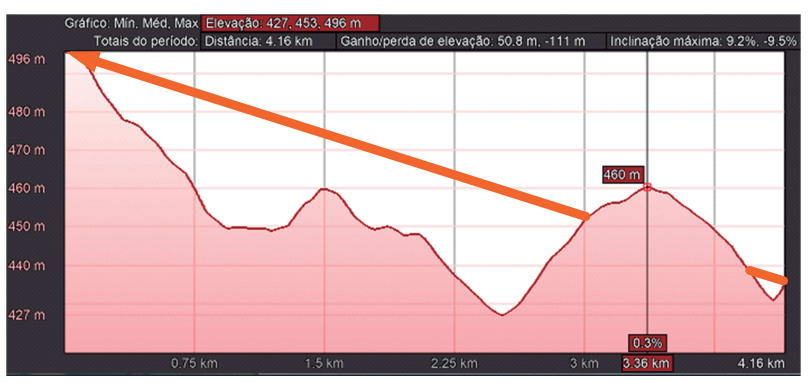

(b)

Fig. 6. Perfil de elevação de um ponto situado a a) $8 \mathrm{~km} \mathrm{e} \mathrm{b)} 4 \mathrm{~km}$ do Gateway.

Packet Error Rate (PER). Além disso, nós queremos finalizar a implantação do sistema para atender às outras funcionalidades na zona rural.

\section{AgRADECIMENTOS}

Os autores gostariam de agradecer a bolsa CNPq PIBIC cota 2020-2021 pelo suporte financeiro à pesquisa.

[6] A. Augustin, J. Yi, T. Clausen, and W. M. Townsley, "A study of lora: Long range amp; low power networks for the internet of things," Sensors, vol. 16, no. 9, 2016. [Online]. Available: https://www.mdpi.com/1424-8220/16/9/1466

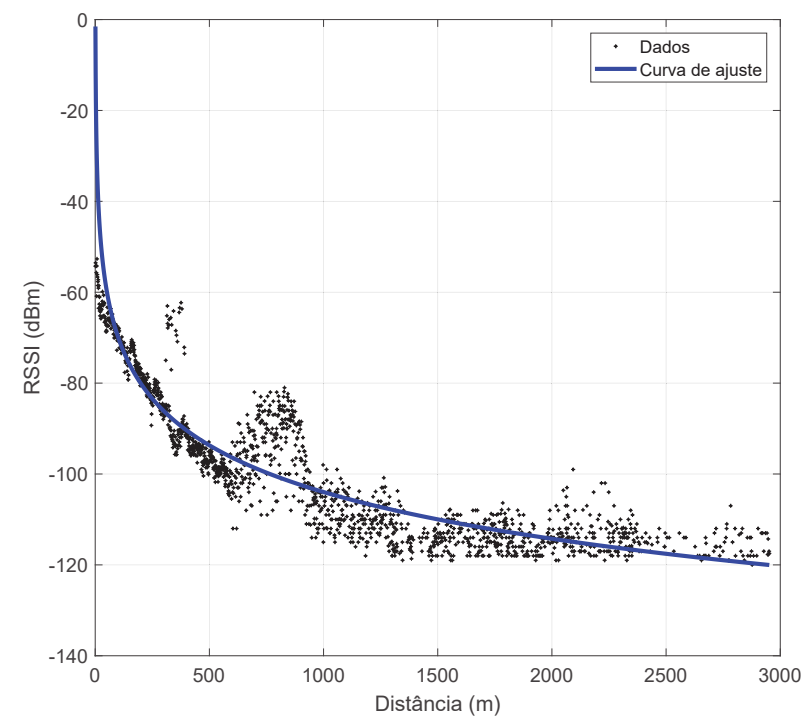

Fig. 7. Ajuste de curva exponencial aos dados.

\section{REFERÊNCIAS}

[1] J. B. S. et al., Indústria 4.0: conceitos e fundamentos. Blucher, 2018.

[2] J. P. Coelho and J. R. Da Silva, Inovação e Tecnologia na Formação Agrícola - Agricultura de Precisão. AJAP, 2009.

[3] J. Han and J. Wang, "An enhanced key management scheme for lorawan," Cryptography, vol. 2, no. 4, 2018. [Online]. Available: https://www.mdpi.com/2410-387X/2/4/34

[4] Anatel, "Ato no 14448, de 04 de dezembro de 2017," 2017. [Online]. Available: https://informacoes.anatel.gov.br/legislacao, 2017.

[5] I. Drotar, B. Lukacs, and M. Kuczmann, "Lorawan network performance test," Acta Technica Jaurinensis, vol. 13, no. 4, p. 268-280, Aug. 2020. [Online]. Available: https://acta.sze.hu/index.php/acta/article/view/547

[7] J. Petajajarvi, K. Mikhaylov, A. Roivainen, T. Hanninen, and M. Pettissalo, "On the coverage of LPWANs: range evaluation and channel attenuation model for LoRa technology," in International Conference on ITS Telecommunications, 2015, pp. 55-59.

[8] D. Yim, J. Chung, Y. Cho, H. Song, D. Jin, S. Kim, S. Ko, A. Smith, and A. Riegsecker, "An experimental lora performance evaluation in tree farm," in 2018 IEEE Sensors Applications Symposium (SAS), 2018, pp. 1-6.

[9] L. F. Ugarte, M. C. Garcia, E. O. Rocheti, E. Lacusta, L. S. Pereira, and M. C. de Almeida, "LoRa communication as a solution for real-time monitoring of IoT devices at UNICAMP," in IEEE International Conference on Smart Energy Systems and Technologies, 2019, pp. 1-6.

[10] T. S. Rappaport, Wireless Communications Principles and Practice. 2nd Edition: Prentice HaD PTR, 2002.

[11] R. R. et al., LPWAN Technologies for IoT and M2M Applications, 2020.

[12] D. W. Marquardt, "An algorithm for least-squares estimation of nonlinear parameters," SIAM Journal on Applied Mathematics, vol. 11, no. 2, pp. 431-441, 1963. 